\title{
Multifocal Central Serous Chorioretinopathy Associated with Steroids in a Patient with Myasthenia Gravis
}

\author{
Maria Stefaniotou Eleni Vourda Andreas Katsanos Miltiadis Aspiotis \\ University Eye Clinic of Ioannina, Ioannina, Greece
}

\section{Key Words}

Low-fluence photodynamic therapy - Ranibizumab - Anti-vascular endothelial growth factor . Subretinal fibrin $\cdot$ Central serous chorioretinopathy treatment

\begin{abstract}
We present a case of bilateral multifocal central serous chorioretinopathy in a 40-year-old male who suffered from myasthenia gravis and was receiving oral prednisolone. Due to the severity of the underlying disease, it was not possible to reduce the corticosteroid dose. After initial unsuccessful treatment with an intravitreal injection of ranibizumab, low-fluence photodynamic therapy was performed, followed by gradual tapering of the corticosteroids. Visual acuity improved significantly in both eyes. Different therapeutic approaches are discussed.
\end{abstract}

\section{Introduction}

Central serous chorioretinopathy (CSCR) is a condition characterized by the accumulation of subretinal fluid at the posterior pole of the fundus, causing single or multiple areas of serous retinal detachment. The pathophysiology of the disease is thought to involve retinal pigment epithelial (RPE) dysfunction, increased leakage of choriocapillaris vessels and Bruch's membrane dysfunction $[1,2]$. The result of localized exudation is the formation of retinal edema and small RPE detachments at the macular and perimacular areas. Although the aetiology and triggering factors of CSCR are not clearly understood, the condition is commonly associated with the use of systemic or topical steroids [2, 3]. Consequently, a critical therapeutic step is the withdrawal of any steroids the patient might be using. 
Unfortunately, when steroids are indispensable for the treatment of a patient's systemic disease, the management of CSCR becomes challenging. Here, we report our experience in such a clinical setting.

\section{Case Report}

A 40-year-old white male was referred to our clinic due to a 15-day history of bilaterally blurred vision and metamorphopsia. He had a history of myasthenia gravis for which he had been treated with oral prednisolone and azathioprine during the last 2 years. His bestcorrected visual acuity (BCVA) was 8/10 in the right eye (OD) and 9/10 in the left eye (OS).

Fundoscopy revealed a few small, yellow-whitish circumscribed macular elevations and one larger, irregularly shaped parafoveal elevation in OD (fig. 1a). Similar small macular lesions were also noted in OS. Fluorescein angiography showed multiple areas of early hyperfluorescence and late leakage in OD (fig. 1b, c). Similar but less pronounced angiography findings were seen in OS (fig. 1d). Optical coherence tomography (OCT) revealed RPE detachment, elevation of the neurosensory retina and highly reflective subretinal fluid mostly in OD (fig. 1e).

The diagnosis of multifocal CSCR was made. As the patient's myasthenia could not be optimally controlled at the time, the treating neurologist advised against prednisolone discontinuation. Unfortunately, we noticed further deterioration of the BCVA and subfoveal expansion of the subretinal fluid in OD, so it was decided to administer an intravitreal injection of ranibizumab in this eye. One month later, his BCVA had deteriorated to 3/10 in OD and $7 / 10$ in OS. Fundoscopy revealed that the area below the fovea in OD was enlarged with the deposition of material presumed to be fibrin in the subretinal space. OCT demonstrated further elevation of the neurosensory retina and a more irregular subretinal accumulation of highly reflective material (fig. 2). Indocyanine green angiography excluded neovascularization and polyp-like vascular abnormalities. Low-fluence photodynamic therapy (PDT) was performed. We administered verteporfin i.v. at a dose of $6 \mathrm{mg} / \mathrm{m}^{2}$ over $10 \mathrm{~min}$, waited for $5 \mathrm{~min}$ and then directed the diode laser at the larger lesion of leakage in OD, using a wavelength of $689 \mathrm{~nm}$ and laser energy of $25 \mathrm{~mJ} / \mathrm{cm}^{2}$. After the first application, the laser spot was directed at the second and the third lesion of leakage in OD (fig. 3a). As the patient's myasthenic symptoms improved, it became possible for the attending neurologist to reduce the dose of prednisolone, increase the dose of azathioprine and add neostigmine. One month later, and while undergoing prednisolone tapering, his BCVA in OD had improved to 6/10 with no subretinal fluid, while the area with the fibrin deposition had nearly disappeared (fig. 3b). The BCVA in OS improved to 10/10 over the next couple of months. To date, 1 year after treatment, the patient's BCVA remains stable at 6/10 in OD and 10/10 in OS with no signs of active disease. The patient's myasthenia is currently well controlled with azathioprine and neostigmine.

\section{Discussion}

Although the aetiology and triggering factors of CSCR are not clearly understood, the condition has been associated with the use of steroids through virtually any route of administration. Furthermore, type A personality, emotional stress, endogenous hypercortisolism as well as an imbalance between sympathetic and parasympathetic activity have also been suggested as risk factors for CSCR $[2,4-6]$. 
Stefaniotou et al:: Multifocal Central Serous Chorioretinopathy Associated with Steroids in a Patient with Myasthenia Gravis

Defective choroidal perfusion and RPE dysfunction are almost invariably present in eyes with CSCR. Corticosteroids may be implicated in these changes by virtue of their propensity to change RPE ionic pump function or alter the permeability of ocular barriers [7]. In addition, steroids increase platelet aggregation and may favor conditions promoting microthrombus formation and increased blood viscosity, thus altering choroidal microcirculation [1].

The suspension of steroids in patients with CSCR is a critical therapeutic intervention. Nevertheless, as shown in our case, the competing interests of continuing steroids for a systemic condition versus the necessity to withhold them in patients with CSCR can lead to therapeutic dilemmas and may call for close cooperation between clinicians of different specialties. In our patient, PDT was helpful in circumventing this therapeutic dilemma. PDT with verteporfin acts by decreasing choroidal permeability and tightening the blood-retinal barrier at the level of the retinal pigment epithelium [8]. Lately, it has been suggested that reducing the light dose (fluence) is safer because standard PDT may cause severe choroidal ischaemia [9], while low-fluence PDT minimizes the risk of Bruch's membrane rupture. Intravitreal anti-vascular endothelial growth factor administration is an alternative approach to CSCR management [10] which has been recently suggested. It is believed that anti-vascular endothelial growth factor exerts its effects by reducing the permeability of the choriocapillaris vessels and tightening the blood-retina barrier. Other therapies that have been proposed include the use of focal argon laser photocoagulation targeted at the leakage sites, micropulse diode laser photocoagulation, transpupillary thermotherapy and corticosteroid antagonists, such as mifepristone and ketoconazole [1, 11-13]. Notwithstanding the various treatment modalities, the discontinuation of any exogenous steroids remains the cornerstone of CSCR treatment.

In our case, as discontinuation of prednisolone was initially contraindicated, it was first decided to proceed with an intravitreal injection of ranibizumab. Contrary to previous reports, however, the result was disappointing as the patient's BCVA continued to decline $[10,14]$. Another possible therapeutic option in our patient was the use of focal argon laser coagulation at sites of leakage [1]. This treatment can be a very useful choice in patients with lesions located away from the fovea. However, in this particular case, it was not attempted because it was thought that laser photocoagulation would entail a high risk of rupture of these very prominent pigment epithelial detachments. In addition, it was felt that photocoagulation through the presumed fibrin that had accumulated in the subretinal space would be of questionable effectiveness.

After performing low-fluence PDT and tapering the steroids, the patient's condition improved dramatically in OD. Interestingly, the clinical picture and BCVA in OS had also markedly improved over a period of a few months without any intervention other than steroid withdrawal. It should be noted that the improvement in OD could, in principle, be attributed to the discontinuation of prednisolone, rather than PDT. We believe, however, that PDT was essential in our case because the resolution of CSCR in OD was immediate, whereas the resolution in OS occurred over the course of some weeks. Additionally, the BCVA achieved immediately after PDT remained practically unchanged during our patient's 1-year follow-up; this indicates that the maximal therapeutic benefit for OD should be attributed to PDT.

It is worth mentioning that the existence of yellow-whitish hyperreflective subretinal material presumed to be fibrin is typically associated with severe forms of CSCR and may signify a poor prognosis [15]. Luckily, our patient's final BCVA in OD $(6 / 10)$ was only moderately reduced compared to the BCVA at presentation (8/10). Our report indicates that 


\begin{tabular}{l|l}
\hline Case Rep Ophthalmol 2013;4:1-6 \\
\hline DOI: $10.1159 / 000351856$ & $\begin{array}{l}\text { C 2013 S. Karger AG, Basel } \\
\text { www.karger.com/cop }\end{array}$ \\
\hline
\end{tabular}

Stefaniotou et al.: Multifocal Central Serous Chorioretinopathy Associated with Steroids in a Patient with Myasthenia Gravis

low-fluence PDT may be helpful when immediate steroid withdrawal is not an option in patients with CSCR.

\section{Disclosure Statement}

The authors have not received grant support or research funding and they do not have any proprietary interests in the materials described in the article. This work has not been presented at any meeting.

\section{References}

1 Gemenetzi M, Salvo GD, Lotery AJ: Central serous chorioretinopathy: an update on pathogenesis and treatment. Eye 2010;24:1743-1756.

-2 Bouzas EA, Karadimas P, Pournaras CJ: Central serous chorioretinopathy and glucocorticoids. Surv Ophthalmol 2002;47:431-448

3 Haimovici R, Koh S, Gagnon DR, et al: Risk factors for central serous chorioretinopathy: a case control study. Ophthalmology 2004;111:244-249.

-4 Yannuzzi LA: Type-A behavior and central serous chorioretinopathy. Retina 1987;7:111-131

-5 Tewari HK, Gadia R, Kumar D, Venkatesh P, Garg SP: Sympathetic-parasympathetic activity and reactivity in central serous chorioretinopathy: a case-control study. Invest Ophthalmol Vis Sci 2006;47:3474-3478.

-6 Bilateral multifocal central serous chorioretinopathy in endogenous hypercortisolism. Clin Exp Optom 2011;94:598-599.

-7 Zamir E: Central serous chorioretinopathy associated with adrenocorticotrophic hormone therapy. A case report and hypothesis. Graefes Arch Clin Exp Ophthalmol 1997;235:339-344.

-8 Chan WM, Lam DS, Lai TY, Tam BS, Liu DT, Chan CK: Choroidal vascular remodelling in central serous chorioretinopathy after indocyanine green angiography guided photodynamic therapy with verteporfin: a novel treatment at the primary disease level. Br J Ophthalmol 2003;87:1453-1458.

-9 Lee PY, Kim KS, Lee WK: Severe choroidal ischaemia following photodynamic therapy for pigment epithelial detachment and chronic central serous chorioretinopathy. Jpn J Ophthalmol 2009;53:52-56.

10 Niegel MF, Schrage NF, Christmann S, Degenring RF: Intravitreal bevacizumab for chronic central serous chorioretinopathy (in German). Ophthalmologe 2008;105:943-945.

-11 Shukla D, Kolluru C, Vignesh TP, Karthikprakash S, Kim R: Transpupillary thermotherapy for subfoveal leaks in central serous chorioretinopathy. Eye 2008;22:100-106.

$\$ 12$ Chen SN, Hwang JF, Tseng LF, Lin CJ: Subthreshold diode micropulse photocoagulation for the treatment of chronic central serous chorioretinopathy with juxtafoveal leakage. Ophthalmology 2008;115:2229-2234.

13 Ross A, Ross AH, Quresh M: Review and update of central serous chorioretinopathy. Curr Opin Ophthalmol 2011;22:166-173.

14 Symeonidis C, Kaprinis K, Manthos K, Androudi S, Anastassilakis K, Dimitrakos SA: Central serous chorioretinopathy with subretinal deposition of fibrin-like material and its prompt response to ranibizumab injections. Case Rep Ophthalmol 2011;2:59-64.

-15 Schatz H, McDonald HR, Johnson RN, Chan CK, Irvine AR, Berger AR, et al: Subretinal fibrosis in central serous chorioretinopathy. Ophthalmology 1995;102:1077-1088. 
Case Reports in

Ophthalmology
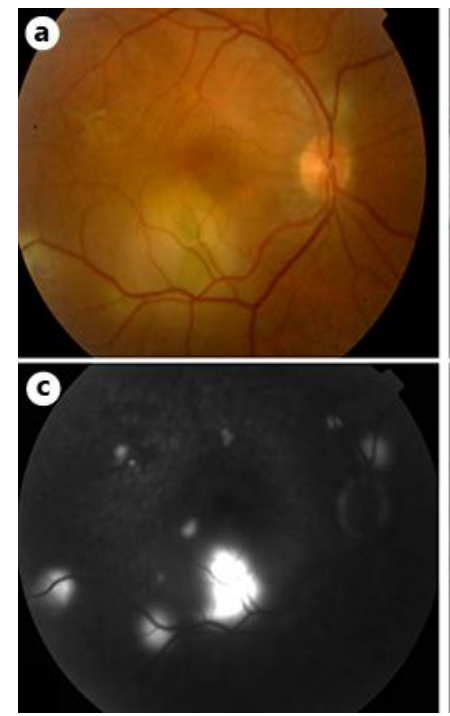

Case Rep Ophthalmol 2013;4:1-6

\section{DOI: $10.1159 / 000351856$}

Stefaniotou et al.: Multifocal Central Serous Chorioretinopathy Associated with

Steroids in a Patient with Myasthenia Gravis

Fig. 1. a Fundus photograph of OD showing multiple macular lesions. b, c Fluorescein angiography of OD showing multiple areas of early hyperfluorescence and late leakage. d Fluorescein angiography of OS showing late leakage. e OCT of OD depicting pigment epithelial detachment and neuroretinal elevation.
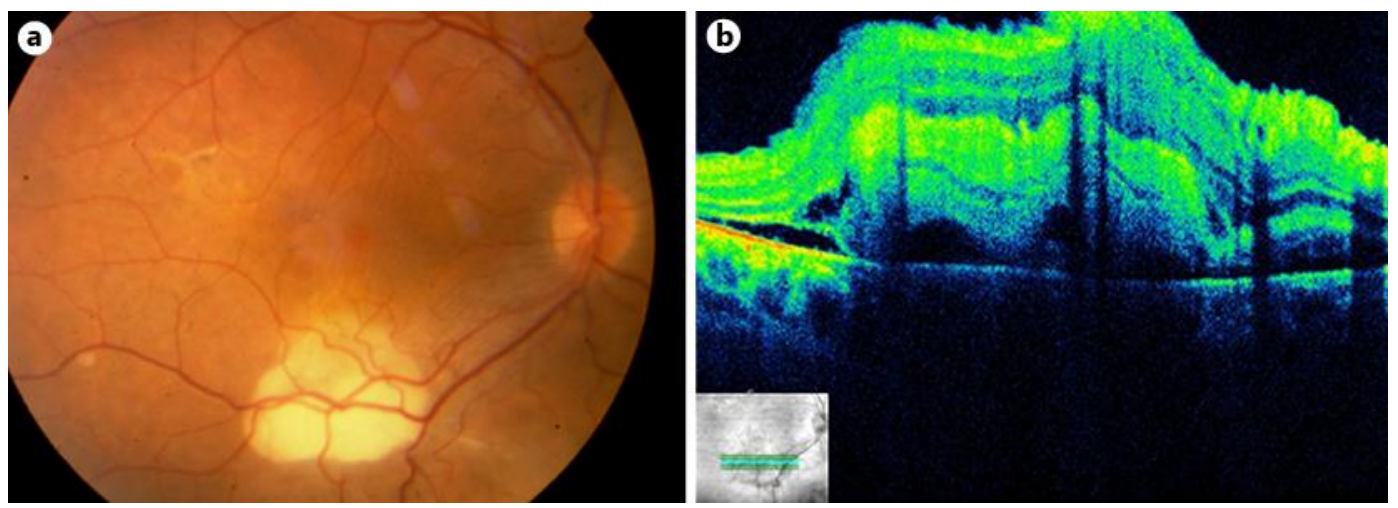

Fig. 2. a Fundus image of OD 1 month after intravitreal ranibizumab. b OCT of OD depicting exacerbation of the retinal elevation with highly reflective subretinal material. 
Case Reports in

Ophthalmology

\begin{tabular}{l|l}
\hline Case Rep Ophthalmol 2013:4:1-6 & \\
\hline DOI: 10.1159/000351856 & $\begin{array}{l}\text { ○ 2013 S. Karger AG, Basel } \\
\text { www.karger.com/cop }\end{array}$ \\
\hline
\end{tabular}

Stefaniotou et al.: Multifocal Central Serous Chorioretinopathy Associated with Steroids in a Patient with Myasthenia Gravis
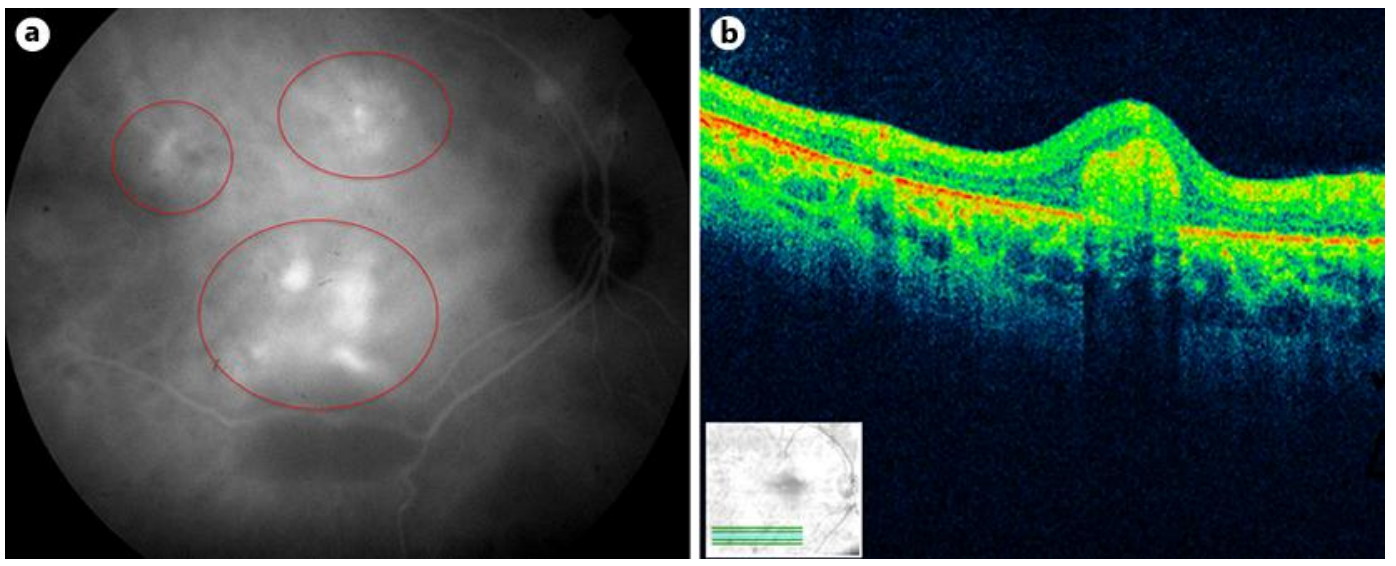

Fig. 3. a Indocyanine green angiography of OD showing the 3 areas of leakage (circled in red) that guided PDT. b OCT of OD documenting regression of the subretinal material. 